\title{
EL RIESGO DE CONTAMINACIÓN POR OZONO EN DOS CIUDADES ESPAÑOLAS (MADRID Y SEVILLA). UN ESTUDIO REALIZADO CON TÉCNICAS DE MODELADO ESPACIAL Y SIG
}

\author{
María Rosa Cañada Torrecilla \\ Universidad Autónoma de Madrid, Ciudad Universitaria de Cantoblanco, \\ C/ Francisco Tomas y Valiente, 1, 28049 Madrid \\ rosa.canada@uam.es
}

\begin{abstract}
Resumen: El ozono troposférico es uno de los contaminantes que más incide en la salud de las personas y alcanza valores elevados en muchas ciudades españolas durante períodos de alta insolación y elevadas temperaturas. De ahí el interés de analizar su distribución espacial en dos ciudades españolas, Madrid y Sevilla. Dada la escasez de información disponible se ha planteado un doble enfoque. Por un lado, analizar los datos registrados por las estaciones de medición y por otro, los datos estimados mediante interpolación espacial, para conocer la contaminación en todo el espacio urbano. La técnica empleada ha sido la media ponderada por el inverso de la distancia (IDW) por lograr los mejores resultados en cuanto a grado de confianza y criterios estadísticos y geográficos. Los resultados alcanzados muestran que las zonas suburbanas o rurales son las más contaminadas por ozono, constituyendo espacios de riesgo para la población, mientras que en el centro de las ciudades los valores de ozono son mucho más bajos.
\end{abstract}

Palabras clave: Contaminación atmosférica, $\mathrm{O}_{3}$, interpolación espacial, SIG, Madrid, Sevilla.

The risk of ozone pollution in two spanish cities (Madrid and Seville). A study performed with spatial modelling and GIS techniques

\begin{abstract}
Tropospheric ozone is one of the pollutants that most affects people's health and reaches high levels in many Spanish cities during periods of high sunshine and high temperatures. Hence the interest in analyzing its spatial distribution in two Spanish cities, Madrid and Seville.
\end{abstract}

Recibido: 28-01-2021. Aceptado: 02-03-2021. 
Given the scarcity of available information, a double approach has been proposed. On the one hand, analyze the data recorded by the measurement stations and on the other, the data estimated by spatial interpolation, to know the pollution in the entire urban space. The technique used was the mean weighted by the inverse of the distance (IDW) to achieve the best results in terms of degree of confidence and statistical and geographical criteria. The results obtained show that suburban or rural areas are the most polluted by ozone, constituting areas of risk for the population, while in the center of cities the ozone values are much lower.

Keywords: Air pollution, $\mathrm{O}_{3}$, Spatial interpolation, GIS, Madrid, Seville.

\section{Introducción}

La contaminación atmosférica es la presencia en el aire de materias o sustancias que son perjudiciales para las personas y el medio ambiente. Muchas de estas sustancias se emiten de manera directa a la atmósfera y otras son el resultado de complejas reacciones químicas. La contaminación es un problema de alcance local, paneuropeo y hemisférico. Los contaminantes emitidos en un país pueden transportarse en la atmósfera y reducir la calidad del aire de otros países.

De todos los contaminantes uno de los más problemáticos para la contaminación urbana y por lo tanto para la salud de sus habitantes es el ozono troposférico $\left(\mathrm{O}_{3}\right)$. Entre 2015-2017 la población residente en zonas urbanas de Europa expuesta a concentraciones atmosféricas de $\mathrm{O}_{3}$ superiores a los límites fijados por la UE ( 8 horas $120 \mu \mathrm{g} / \mathrm{m}^{3}$ ), alcanza porcentajes que oscilan entre el 12 y el 29\%, si se considera el umbral de la OMM (8 horas $100 \mu \mathrm{g} / \mathrm{m}^{3}$ ) los porcentajes fluctúan entre 95-98\% (EEA, 2019).

El ozono troposférico puede ser de origen natural o producto de las actividades humanas. De manera natural, puede proceder de modo directo de intrusiones del ozono de la estratosfera (Song et al., 2011), de las descargas eléctricas de las tormentas, que alteran el oxígeno atmosférico, o de emisiones procedentes de actividades naturales, como la vegetación, los volcanes y las fermentaciones (Díaz y Linares, 2005). Pero la principal fuente del ozono troposférico es la de origen antropogénico, siendo en este caso un contaminante secundario, que se forma en la atmósfera mediante una serie de reacciones químicas entre contaminantes primarios, llamados precursores, como los óxidos de nitrógeno (NOx) y los compuestos orgánicos volátiles (COV) en unas condiciones de alta temperatura e insolación (Díaz y Linares, 2005).

La mayoría de los NOx existentes en el aire de las ciudades son de origen antrópico y proceden de la combustión de materiales orgánicos tanto en fuentes estaciona- 
rias (calefacciones, procesos industriales y centrales térmicas) como en fuentes móviles (vehículos de gasolina y diésel). Los COV están fundamentalmente constituidos por hidrocarburos (benceno, tolueno), por combustión incompleta de éstos (alcanos, alquenos, aromáticos, alcoholes y algunos compuestos clorados). También influyen en la formación de ozono compuestos inorgánicos como el CO, muy presente en atmósferas contaminadas.

De manera simplificada, el proceso de producción del ozono se inicia al reaccionar los óxidos de nitrógeno con luz solar de longitud de onda inferior a $400 \mathrm{~nm}$ (1 nm $=10^{-9} \mathrm{~m}$ ), según el siguiente proceso: $\mathrm{NO}_{2}+$ luz $\mathrm{NO}+\mathrm{O}$, el oxígeno atómico así formado reacciona rápidamente con el oxígeno del aire para formar ozono, $\mathrm{O}+\mathrm{O}_{2} \mathrm{O}_{3}$. Por otro lado, en entornos urbanos contaminados el NO recién emitido puede combinarse inmediatamente con el ozono reduciendo sus concentraciones en el ambiente, según la siguiente reacción: $\mathrm{NO}+\mathrm{O}_{3} \mathrm{NO}_{2}+\mathrm{O}_{2}$. Esto hace que normalmente los máximos de ozono no se den en el centro de las ciudades sino en los parques y en la periferia de estas, donde son menores las emisiones a la atmósfera de NOx. Debido a este proceso, una reducción de las emisiones de NOx en las ciudades puede dar lugar a un aumento de las concentraciones de ozono. En Europa, de manera global, las emisiones de los precursores del ozono, NOx y COV están descendiendo y en consecuencia los niveles de ozono en las ciudades se están incrementando, aunque también son consecuencia de la tendencia al incremento de las temperaturas medias y de las situaciones meteorológicas extremas (olas de calor) resultado del cambio climático.

No se debe olvidar, además, el papel que tiene el ozono troposférico como gas de efecto invernadero ya que absorbe la radiación de onda larga y contribuye al calentamiento del planeta (EEA, 2014).

Las concentraciones de ozono muestran un característico ciclo diario con mínimas concentraciones a primeras horas de la mañana y máximas a primeras horas de la tarde (Dueñas et al., 2002). En íntima relación con la actividad fotoquímica a lo largo del día, es más favorecida cuando hay inversión térmica junto al suelo y los vientos son suaves (Saavedra et al., 2012).

La polución es el riesgo ambiental más importante por sus efectos sobre la salud humana. Los ciudadanos europeos lo perciben como el segundo riesgo ambiental más importante tras el cambio climático (European Comission, 2017). El ozono es un potente oxidante y, por tanto, es extremadamente irritante para el sistema respiratorio. Además, el ozono es menos soluble que otros gases irritantes, y puede penetrar más eficazmente a través del árbol traqueobronquial induciendo lesiones en las células del pulmón. Las respuestas del tracto respiratorio inducidas por el ozono incluyen reducción en la función pulmonar y el empeoramiento de enfermedades preexistentes, como asma. Cuando una persona realiza sus actividades físicas al aire libre respira más rápido y profundo con lo que aumenta la dosis de contaminante recibida y la mayor penetración del ozono a regiones más profundas del pulmón. 
También puede producir otros síntomas como irritación de los ojos, dolor de cabeza, dolor de pecho, tos, etc.

Estudios recientes han establecido un nexo entre el ozono y patologías cardiovasculares. El ozono provoca una constricción arterial, disminuyendo por tanto el diámetro de las arterias y aumentando la posibilidad de afecciones relacionadas con procesos vasculares.

La ola de calor del año 2003 en Europa ha puesto de manifiesto la existencia de una clara asociación entre altas temperaturas y niveles máximos de ozono. Estudios realizados en Francia, Bélgica y Suiza indican que una parte de la mortalidad atribuible a la ola de calor estuvo relacionada con los altos niveles de ozono registrados (Diaz y Linares, 2005).

Según un estudio epidemiológico realizado para la ciudad de Madrid existe una alta relación entre el ozono y la mortalidad e ingresos hospitalarios. Según este trabajo existe una concentración media diaria que marca un umbral a partir del cual concentraciones superiores comienzan a tener influencia sobre la morbi-mortalidad. Este umbral, que en ambos casos está próximo a los $40 \mu \mathrm{g} / \mathrm{m}^{3}$, muestra que un incremento de 25 $\mu \mathrm{g} / \mathrm{m}^{3}$ sobre este nivel se traduce en un aumento de un $12 \%$ en la mortalidad y en un $18 \%$ para el caso de los ingresos por urgencias tanto por patologías respiratorias como cardiovasculares. Estos incrementos son seis veces superiores a los detectados en los contaminantes primarios como las partículas en suspensión o el dióxido de azufre, para el conjunto de la población (Ferrán, Tenías y Pérez-Hoyos, 1999).

Los efectos del ozono sobre el medio ambiente son también importantes. El ozono se encuentra entre los contaminantes atmosféricos más perjudiciales para la vegetación. Se ha observado que los episodios cortos de altas concentraciones producen efectos agudos sobre las plantas, mientras que las exposiciones prolongadas a bajas concentraciones producen efectos de carácter crónico. Los primeros son fácilmente visibles y se aprecian en las hojas mediante manchas de color rojizo, marrón o púrpura. Los efectos crónicos son menos visibles produciendo daños a nivel metabólico. En ambos casos, la acción del ozono reduce la fotosíntesis neta y puede aumentar el envejecimiento prematuro, traduciéndose todo ello en un rendimiento menor de los cultivos (Dingenen et al., 2009; Felzer et al., 2007, Fishman et al., 2010) y en el crecimiento de los bosques (Gottardini et al., 2010, Loibl et al., 2004). El ozono penetra en las hojas de las plantas a través de los estomas, alterando de forma progresiva la integridad de las células, lo que se traduce en la reducción de la fotosíntesis neta en la planta. El ozono también tiene impacto sobre los materiales (EEA, 2015).

Algunos grupos de población se ven más afectados que otros por este contaminante fotoquímico, en unos casos porque están más expuestos, como son los grupos de población de nivel social y económico más bajo, y en otros casos porque son grupos más vulnerables, como las personas mayores, embarazadas o niños (EEA, 2019; Ayuntamiento de Madrid, 2020). 
Ante esas preocupantes evidencias de la relación entre este contaminante y sus efectos sobre la salud humana, nuestro principal objetivo ha sido doble: a) determinar y sintetizar el patrón espacial del $\mathrm{O}_{3}$, localizando las zonas que con frecuencia superan los umbrales establecidos por la legislación en las ciudades españolas de Madrid y Sevilla; y b) cuantificar la extensión del territorio y población expuestos a condiciones adversas. Por otra parte, este trabajo viene a rellenar un hueco existente en la investigación de este tema hasta la fecha. En última instancia se busca patentizar ante políticos, gestores y ciudadanos la deficiente calidad del aire urbano y concienciarles para que se adopten medidas legislativas más estrictas y acordes con las recomendaciones de la OMS, encaminadas a mejorar los estándares ambientales y la calidad de la vida urbana.

\section{Materiales y Métodos}

Las ciudades elegidas para este estudio han sido Madrid y Sevilla, con una población de 3.273.049 hab. y 704.198 hab. respectivamente, según datos del INE de 2010. El ámbito de estudio recae exclusivamente en el área urbana poblada (AUP), es decir, en espacio de uso residencial, comercial, ocio, etc. y donde se excluyen las áreas de uso industrial, transporte (e. g. aeropuertos), agrícola, ganadero, forestal, etc. La delimitación de dicha zona se realizó a partir de la interpretación de imágenes aéreas recientes (Plan Nacional de Ortofotografía Aérea del Instituto Geográfico Nacional) y de cartografía de usos del suelo (Corine LandCover, 2006).

Los datos de contaminación proceden en el caso de Madrid de la Red de Vigilancia de la Calidad del Aire del Ayuntamiento de Madrid y de la Comunidad de Madrid, y los de Sevilla de la Red de Vigilancia y Control de la Calidad del Aire de Andalucía. En ambos casos, dado el reducido número de estaciones de medición intraurbanas, se han utilizado estaciones próximas situadas en municipios limítrofes para mejorar la cobertura del muestreo espacial. El número de estaciones han sido 22 en Madrid y 8 en Sevilla.

Los niveles de ozono admisibles por la legislación europea y española se han reflejado en la tabla 1. El indicador adoptado aquí ha sido el valor objetivo de protección a la salud que se establece en el número de días en que se supera el valor de $120 \mu \mathrm{g} / \mathrm{m}^{3}$ como media móvil octohoraria, que no podrá ser superior a 25 días por año en un periodo de tres años. Si se supera ese umbral entraríamos en una zona de riesgo por alta contaminación por ozono en la ciudad. Los tres años utilizados han sido 2010, 2011 y 2012.

La Organización Mundial de la Salud (OMS) en la protección a la salud establece un valor guía mucho más restrictivo si bien no tiene valor legal $\left(100 \mu \mathrm{g} / \mathrm{m}^{3}\right.$ en lugar de $\left.120 \mu \mathrm{g} / \mathrm{m}^{3}\right)$. 
Tabla 1. Niveles admisibles de ozono troposférico

\begin{tabular}{|c|c|c|}
\hline Umbral & Valor & Periodo de referencia \\
\hline Información a la población & $180 \mu \mathrm{g} / \mathrm{m}^{3}$ & Valor medio en una hora \\
\hline Alerta a la población & $240 \mu \mathrm{g} / \mathrm{m}^{3}$ & $\begin{array}{l}\text { Promedio horario. Se evalúa durante } \\
\text { tres horas consecutivas }\end{array}$ \\
\hline Protección a la salud & $120 \mu \mathrm{g} / \mathrm{m}^{3}$ & $\begin{array}{l}\text { Media móvil octohoraria en un día, } \\
\text { que no podrá superarse más de } \\
25 \text { días por año de promedio } \\
\text { en un periodo de tres años }\end{array}$ \\
\hline Protección de los bosques & AOT $40=6000 \mu \mathrm{g} / \mathrm{m}^{3} \mathrm{~h}$ & Valores horarios de mayo a julio \\
\hline Protección a la vegetación & AOT $40=6000 \mu \mathrm{g} / \mathrm{m}^{3} \mathrm{~h}$ & $\begin{array}{l}\text { Valores horarios de abril } \\
\text { a septiembre }\end{array}$ \\
\hline Daños a los materiales & $40 \mu \mathrm{g} / \mathrm{m}^{3}$ & Año civil \\
\hline
\end{tabular}

Fuente: Elaboración propia.

Para conseguir información continua del ozono en todo el espacio urbano (AUP) se ha recurrido a la aplicación de técnicas de interpolación mediante la extensión Geostatistical Analyst de ArcGIS. Se consigue así el modelo espacial del ozono en ambas ciudades, estimando valores de zonas donde no hay información a partir de datos muestrales. En trabajos realizados con anterioridad se han examinado y comparado sistemáticamente los resultados alcanzados con la utilización de los diferentes métodos de interpolación, analizado las diferencias y similitudes que emergen en los patrones espaciales obtenidos con las distintas técnicas, valorado su grado de confianza y dirimido cuáles son los más adecuados, teniendo en cuenta criterios estadísticos y geográficos (Cañada et al., 2014).

Para el ozono el método que mejor refleja el reparto espacial del contaminante es el de la media ponderada por el inverso de la distancia (IDW), en su modalidad anisotrópica, en ambas ciudades, al ajustarse más a la orografía de los municipios y a los vientos dominantes. La dirección de máxima continuidad espacial de la variable marcada por el eje principal de la elipse es NE-SW, con $45^{\circ}$ de inclinación, el número de cuadrantes en que se ha dividido la elipse es de 4 y el número de vecinos utilizados varía entre un máximo de 5 y un mínimo de 2 . El valor de p es 1 (véase tabla 2). Con estos parámetros, aunque no han proporcionado los menores errores, se ha conseguido una distribución espacial del contaminante más compatible y coherente con el marco territorial y la estructura urbana local del municipio. 
Una vez obtenidas las capas estimadas se han transformado a raster con un tamaño de celda de $50 \mathrm{~m}$. Con la extensión Spatial Analyst y mediante el comando Raster calculator, se han recortado para ajustarlas a los límites del área urbana poblada en cada ciudad.

La metodología para calcular la superficie y población expuesta a la contaminación por ozono de cada ciudad se ha desarrollado en varias etapas. En primer lugar, se convirtió a raster la información demográfica, es decir, a las secciones censales de la capa vectorial (recortadas al AUP) se le asociaron con ArcGIS, los datos de población por sección censal y después se rasterizó dicha capa en píxeles de 50 m.

A partir de otra capa raster del AUP, en la que el valor de cada píxel de $50 \mathrm{~m}$ es el código numérico de la sección censal a la que pertenece, se obtuvo una nueva capa raster conteniendo, para cada sección censal, el número de píxeles que poseía dicha sección. Realizando el cociente (calculadora raster) de las dos capas anteriores (de población y de número de píxeles por sección), se consiguió una nueva capa raster con el número de habitantes por píxel, dentro de cada sección censal, es decir, se repartió homogéneamente la población de cada sección censal entre los píxeles que contenía.

Finalmente, con las herramientas de análisis espacial de ArcGIS (Zonal statistics as a table) se relacionaron las capas de contaminantes y de población, obteniendo el recuento en valores absolutos y relativos (respecto del total de cada ciudad) de píxeles (por consiguiente, de superficie) y de población contenidos en cada intervalo de contaminante anteriormente establecido según los umbrales normativos. Es decir, la población expuesta y no expuesta a aire degradado (Cañada, Moreno y Martínez, 2020).

Del mismo modo, a partir de la capa interpolada, con el comando Zonal statístics as table, se realizaron los mapas de distribución del ozono por distritos.

Tabla 2. Parámetros utilizados en la interpolación y bondad de ajuste del modelo retenido

\begin{tabular}{|l|l|c|c|}
\hline \multirow{2}{*}{ Modelo } & \multirow{2}{*}{ Parámetros } & \multicolumn{2}{|c|}{${\text { Contaminante } \mathrm{O}_{3}}^{*}$} \\
\cline { 2 - 4 } & Madrid & Sevilla \\
\hline \multirow{2}{*}{ IDW anisotrópico } & N.o vecinos & $5 / 2$ & $5 / 2$ \\
& Valor p & 1 & 1 \\
& Búsqueda de vecindad & Elipse, 4 partes, & Elipse, 4 partes, \\
& & $45^{\circ}, 9000 / 4000$ & $45^{\circ}, 40000 / 10000$ \\
& Ángulo & 30 & 65 \\
\hline Bondad de ajuste & Media de errores (mean error) & 1,68 & $-0,002$ \\
& Error cuadrático medio (RMS) & 8,52 & 14,4 \\
\hline
\end{tabular}

Fuente: Elaboración propia. 


\section{Análisis de Resultados}

\subsection{Características de la contaminación por ozono troposférico a partir de los datos observados en las estaciones}

Los estadísticos descriptivos de las dos ciudades (tabla 3) indican que el número medio de días en que se superan los $120 \mu \mathrm{g} / \mathrm{m}^{3}$ de ozono asciende a 15 en Madrid y casi 25 en Sevilla. Los valores máximos se alcanzan en Mairena del Aljarafe (46) y en Majadahonda (43), municipios ambos periféricos a Sevilla y a Madrid respectivamente.

Tabla 3. Estadísticos descriptivos del número de días de ozono superiores a $120 \mathrm{~g} / \mathrm{m}^{3}$ (2010-2012), observados en las estaciones de Madrid y Sevilla

\begin{tabular}{|l|c|c|}
\hline Estadisticos descriptivos & Madrid & Sevilla \\
\hline Media & 15,2 & 24,7 \\
\hline Desviación típica & 11,65 & 12 \\
\hline Máximo & 43 & 47,3 \\
\hline Mínimo & 0 & 1 \\
\hline N & 22 & 8 \\
\hline
\end{tabular}

Fuente: Elaboración propia

En Madrid, el mapa de símbolos graduados (figura 1) muestra que son estaciones suburbanas (Juan Carlos I y El Pardo) y estaciones de municipios limítrofes (Alcobendas, Majadahonda) las que rebasan el umbral de los 25 días como promedio entre 20102012, límite fijado por la legislación. La mayoría de las estaciones de tráfico y de fondo urbano registran entre 0 y 3 días. En Sevilla hay cuatro estaciones por encima del umbral legal; además de Mairena del Aljarafe, ya citada, Alcalá de Guadaira, Santa Clara y Centro (figura 2). Este comportamiento se explica porque el ozono es destruido al combinarse con los óxidos de nitrógeno para formar $\mathrm{NO}_{2}$, por eso en aquellas zonas con altas tasas de $\mathrm{NO}_{2}$ hay valores bajos de ozono (Cañada y Moreno, 2017).

\subsection{Características de los datos estimados de $\mathrm{O}_{3}$. Análisis de sus patrones espaciales. Superficie y población expuestas}

Como resultado del modelado del $\mathrm{O}_{3}$ con el método de interpolación, media ponderada por el inverso de la distancia (IDW), se han obtenido unos valores estimados para el área urbana poblada (AUP), cuyos resúmenes estadísticos se han recogido en la tabla 4. La media y el valor máximo estimados de $\mathrm{O}_{3}$ en Madrid y Sevilla son menores que los valores observados, sobre todo en los máximos, donde el recorte es muy importante. 


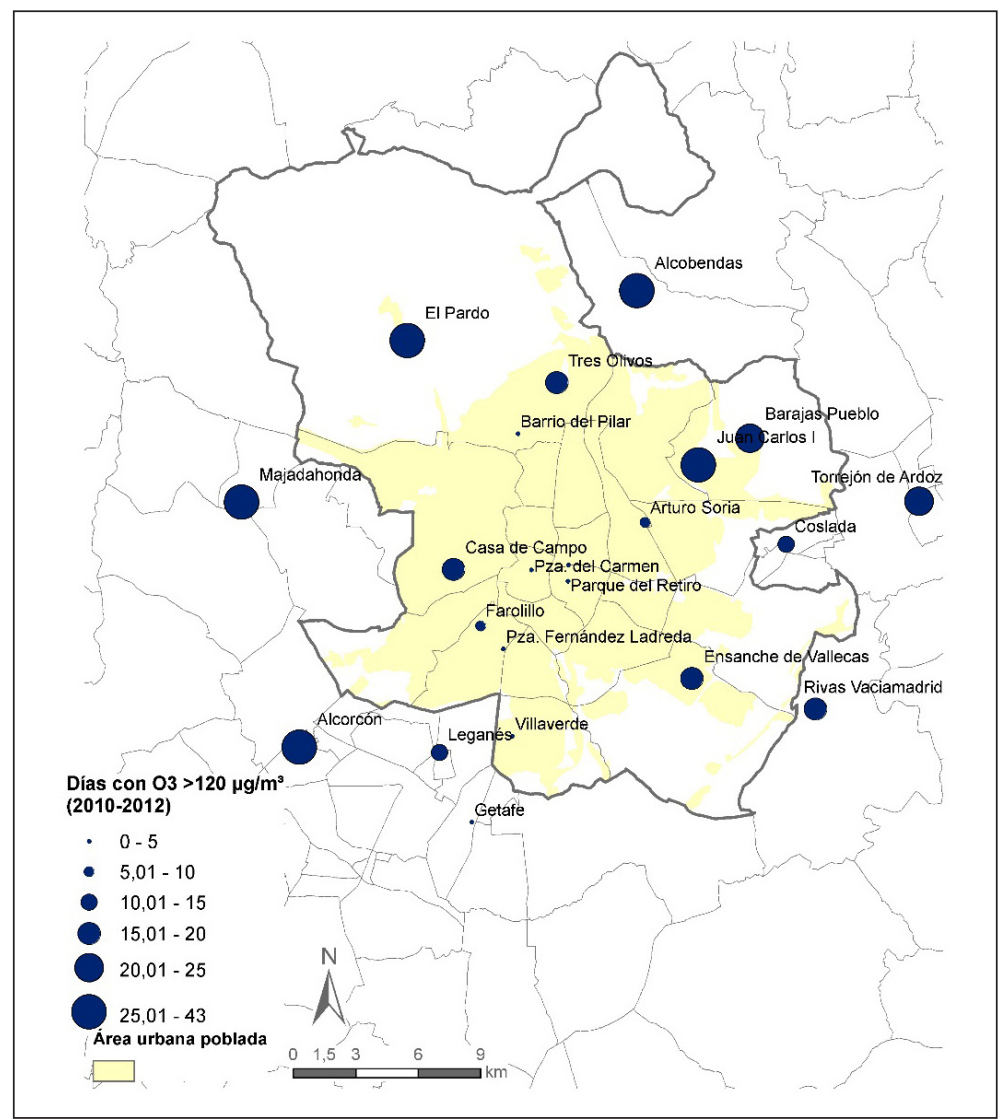

Figura 1. Días con $\mathrm{O}_{3}$ superiores a $120 \mu \mathrm{g} / \mathrm{m}^{3}$ en Madrid (2010-2012). Fuente: Elaboración propia.

Tabla 4. Estadísticos descriptivos de las estimaciones del número de días de ozono superiores a $120 \mathrm{\mu g} / \mathrm{m}^{3}$ (2010-2012) para el AUP de Madrid y Sevilla

\begin{tabular}{|l|c|c|}
\hline Estadisticos descriptivos & Madrid & Sevilla \\
\hline Media & 13,5 & 23,8 \\
\hline Desviación típica & 5,67 & 3,8 \\
\hline Máximo & 33,79 & 30,92 \\
\hline Mínimo & 0,07 & 1,55 \\
\hline Amplitud & 33,73 & 29,37 \\
\hline
\end{tabular}

Fuente: Elaboración propia. 


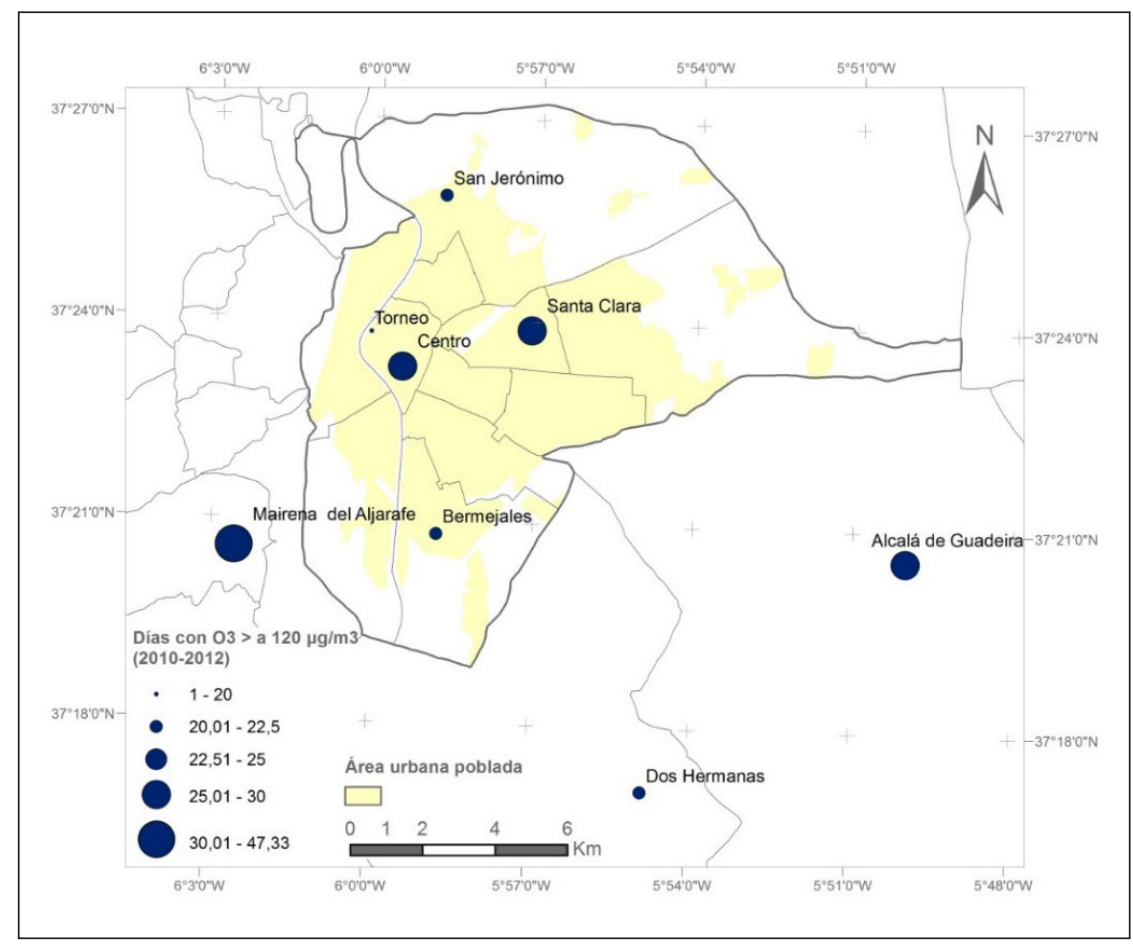

Figura 2. Días con $\mathrm{O}_{3}$ superiores a $120 \mu \mathrm{g} / \mathrm{m}^{3}$ en Sevilla (2010-2012).

Fuente: Elaboración propia.

Los mapas de estimación de $\mathrm{O}_{3}$ realizados con interpolación, se han construido con seis intervalos, diferenciando uno por encima de 25 días, umbral establecido por la legislación, indicaría que se incumple la normativa y, cinco por debajo de ese límite. La mayor parte de la ciudad de Madrid (figura 3) muestra una buena calidad del aire, con cifras inferiores a 25 días en los que se superan los $120 \mu \mathrm{g} / \mathrm{m}^{3}$ (colores verdes sobre el mapa; solamente ciertos ámbitos del noroeste (Aravaca y El Pardo) y noreste (Barajas), el $2 \%$ del AUP (figura 5, tabla 5), presentarían problemas de incumplimiento de la legislación y por lo tanto una atmósfera peligrosa para las personas, plantas y materiales. Sevilla revela una peor calidad del aire en cuanto a contaminación por ozono. El espacio por encima de 25 días representado en color rojo sobre el mapa (figura 4), supone el 57,8\% de la superficie del AUP (figura 5 izda., tabla 5). 


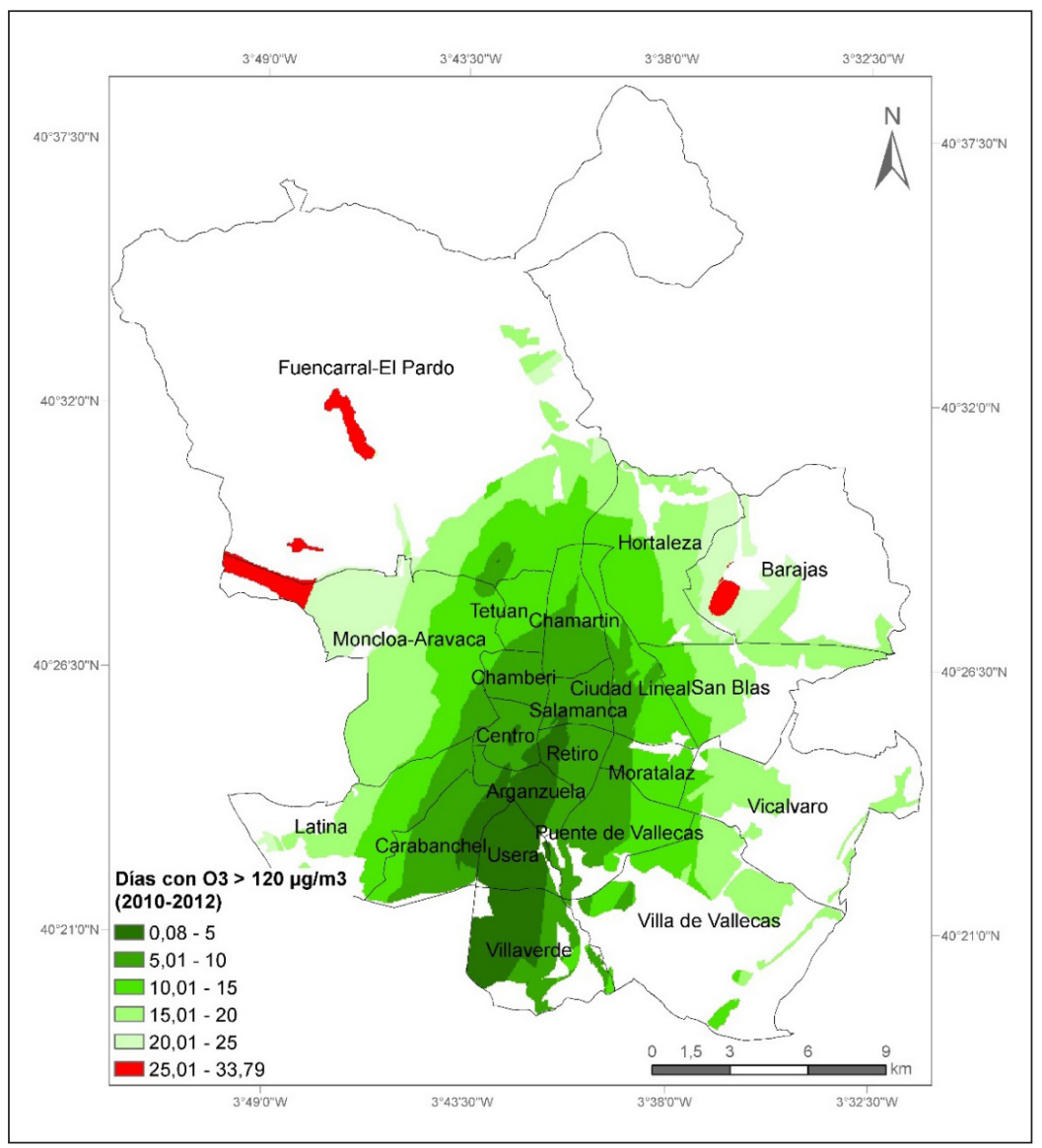

Figura 3. Patrón espacial de la estimación de $\mathrm{O}_{3}$ en la ciudad de Madrid.

Fuente: Elaboración propia.

Tabla 5. Superficie y población de Madrid y Sevilla expuestas a valores de $\mathrm{O}_{3}$ por encima del umbral crítico de 25 días con media octohoraria $>120 \mu \mathrm{g} / \mathrm{m}^{3}$ 2010-2012

\begin{tabular}{|l|c|c|c|c|}
\hline Ciudad & $\begin{array}{c}\text { Área expuesta } \\
\text { (ha) }\end{array}$ & $\begin{array}{c}\text { \% de área } \\
\text { expuesta }\end{array}$ & $\begin{array}{c}\text { Población } \\
\text { expuesta (hab.) }\end{array}$ & $\begin{array}{c}\text { \% de población } \\
\text { expuesta }\end{array}$ \\
\hline Madrid & 590,50 & 2,18 & 6.394 & 0,20 \\
\hline Sevilla & $3.951,50$ & 57,96 & 398.101 & 56,53 \\
\hline
\end{tabular}

Fuente: Elaboración propia. 


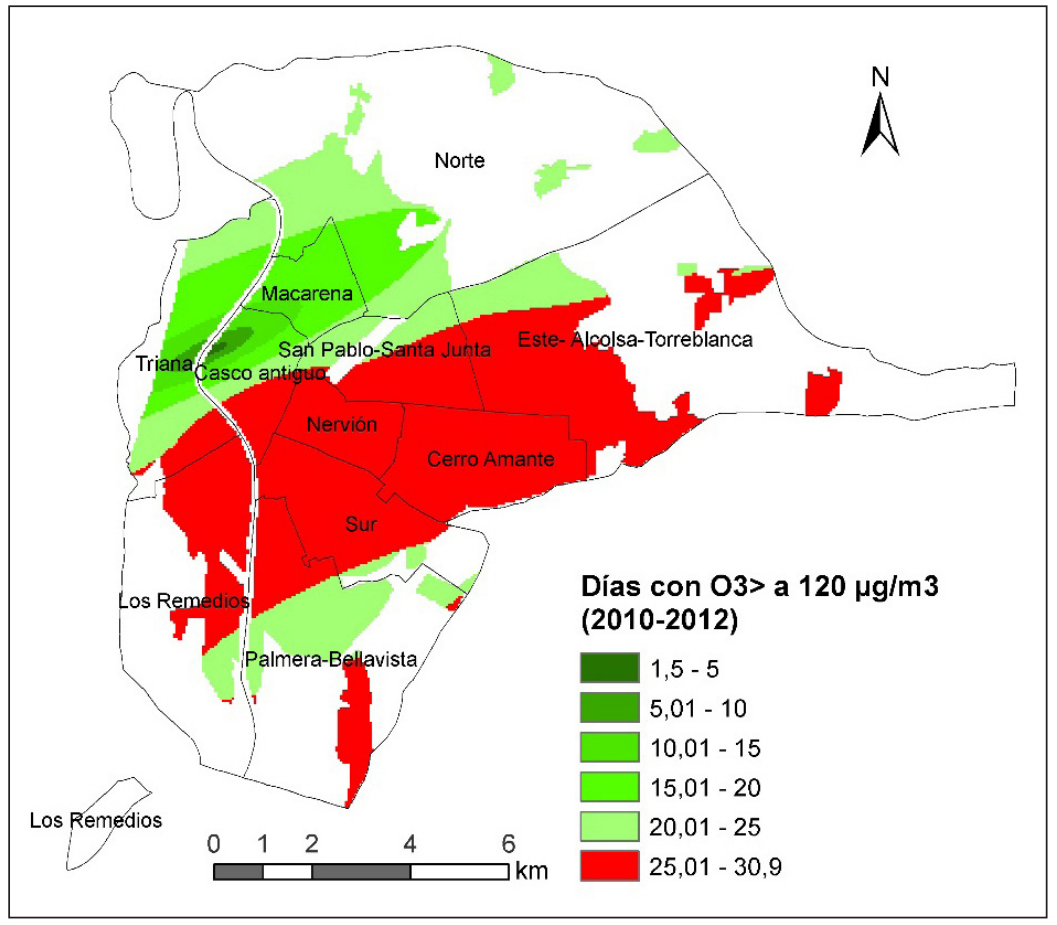

Figura 4. Patrón espacial de la estimación de $\mathrm{O}_{3}$ en la ciudad de Sevilla. Fuente: Elaboración propia.

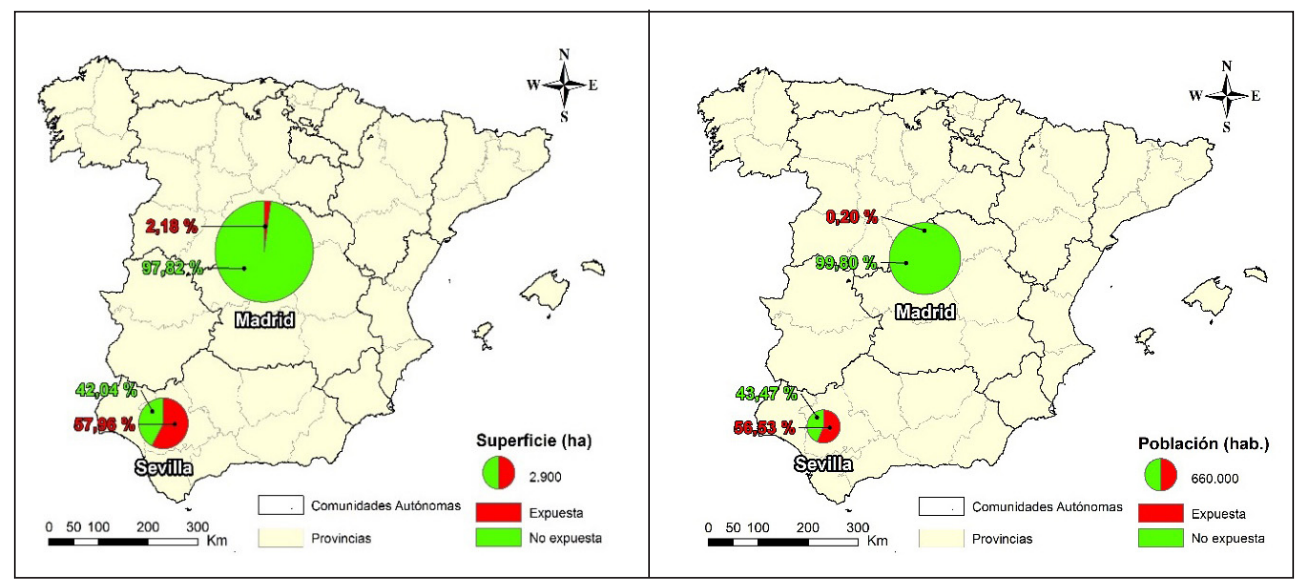

Figura 5. Superficie y población expuesta a $\mathrm{O}_{3}$ alto en Madrid y Sevilla (2010-2012). Fuente: Elaboración propia. 
Factores geográficos, meteorológicos y reacciones fotoquímicas explicarían esta distribución espacial. En ciudades con elevadas concentraciones de óxidos de nitrógeno, éstos destruyen el $\mathrm{O}_{3}$, contribuyendo a su disminución en la atmósfera, por el contrario el ozono permenece en aquellos ambientes donde las emisiones a las atmósfera de NOx son menores.

La población expuesta al contaminante en Madrid alcanza sólo el 0,20\%, mientras que en Sevilla la cifra se eleva al 56,53\% (figura 5 dcha., tabla 5).

\subsection{Análisis de la distribución por distritos}

Los mapas de contaminación por distritos se han realizado con los estadísticos descriptivos calculados a partir de la capa raster obtenida de la interpolación. El parámetro representado es el valor medio de cada distrito. En Madrid (figura 6) al observar la distribución del número de días superiores a $120 \mu \mathrm{g} / \mathrm{m}^{3}$ el único distrito que incumple la legislación es Barajas, porque presenta más de 25 días en que se han superado los $120 \mu \mathrm{g} / \mathrm{m}^{3}$, los restantes no tienen una mala calidad del aire por ozono troposférico.

La ciudad de Sevilla (figura 7) muestra una peor calidad del aire. Son seis distritos, en color rojo, los que superan el umbral de 25 días que es el establecido por la legislación. El resto exhibe valores inferiores (representados por colores verdes sobre el mapa).

\section{Discusión y conclusiones}

En este trabajo se han aplicado técnicas de interpolación espacial para estimar los valores de ozono troposférico en las ciudades de Madrid y Sevilla. Es muy frecuente el empleo de este tipo de métodos como ha puesto de manifiesto Jerrett et al. (2005), si bien ellos citan al kriging como el interpolador más común y el óptimo, porque permite valorar el grado de incertidumbre de los valores estimados. Moral et al. (2012), utilizaron técnicas geoestadísticas para evaluar la distribución de ozono en la ciudad de Badajoz. Aquí se ha preferido la media ponderada por el inverso de la distancia (IDW) debido a la escasez de estaciones, sobre todo en Sevilla, y a que el resultado del modelado espacial se ajusta mejor a la geografía de la ciudad. Palacios et al. (2002) utilizaron modelos dispersión de contaminantes para explicar las concentraciones de ozono en la zona de Madrid y su área metropolitana.

Se ha utilizado como indicador de contaminación el establecido por la legislación, número de días con valores máximos octohorarios superiores a $120 \mu \mathrm{g} / \mathrm{m}^{3}$, que no podrá ser superior a 25 días por año en un periodo de tres años. En otros trabajos han elegido trabajar con valores medios de concentración de ozono y su relación con otras 


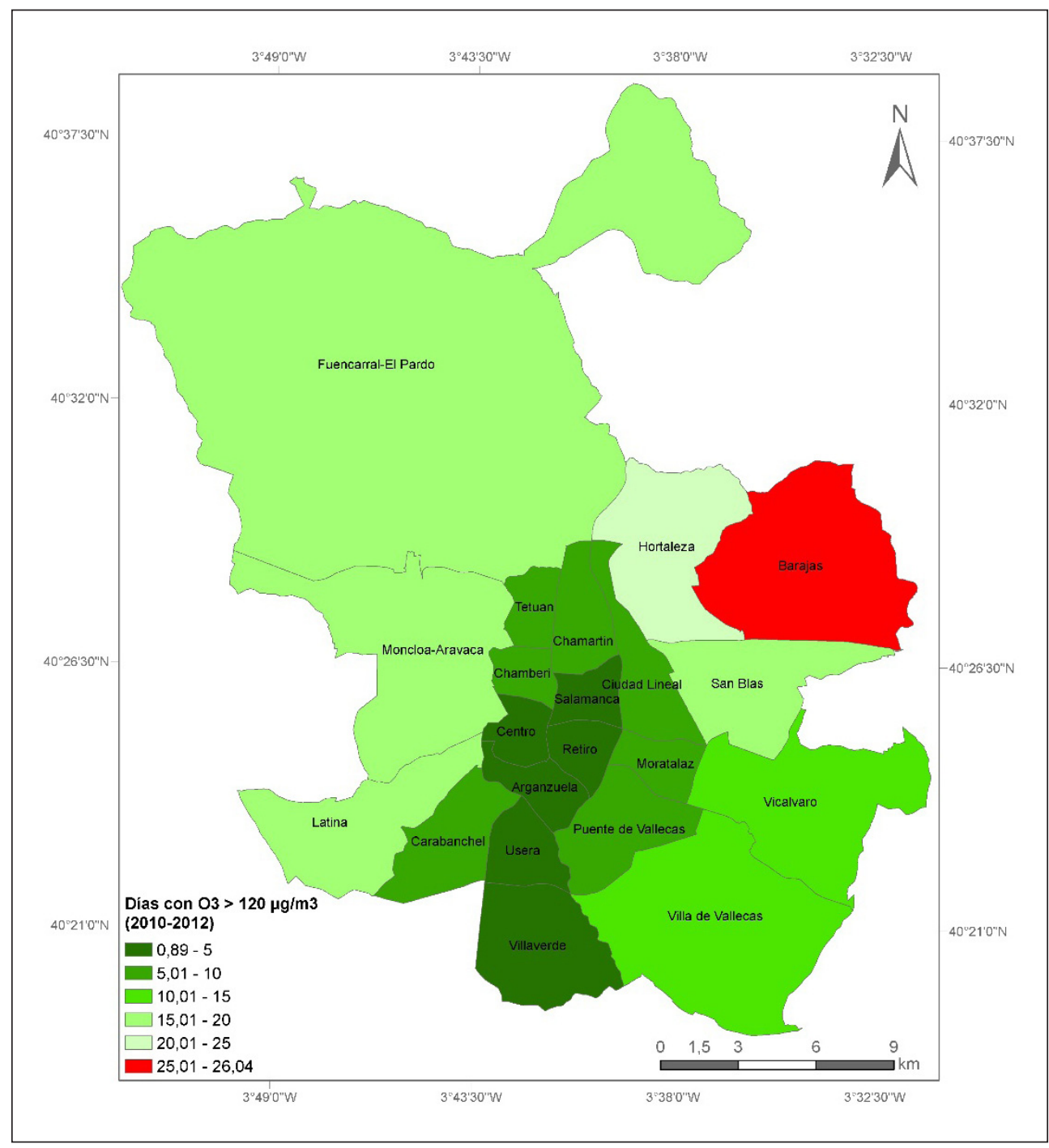

Figura 6. Número de días con $\mathrm{O}_{3}$ superiores a $120 \mu \mathrm{g} / \mathrm{m}^{3}$ en los distritos de Madrid (2010-2012).

Fuente: Elaboración propia. 


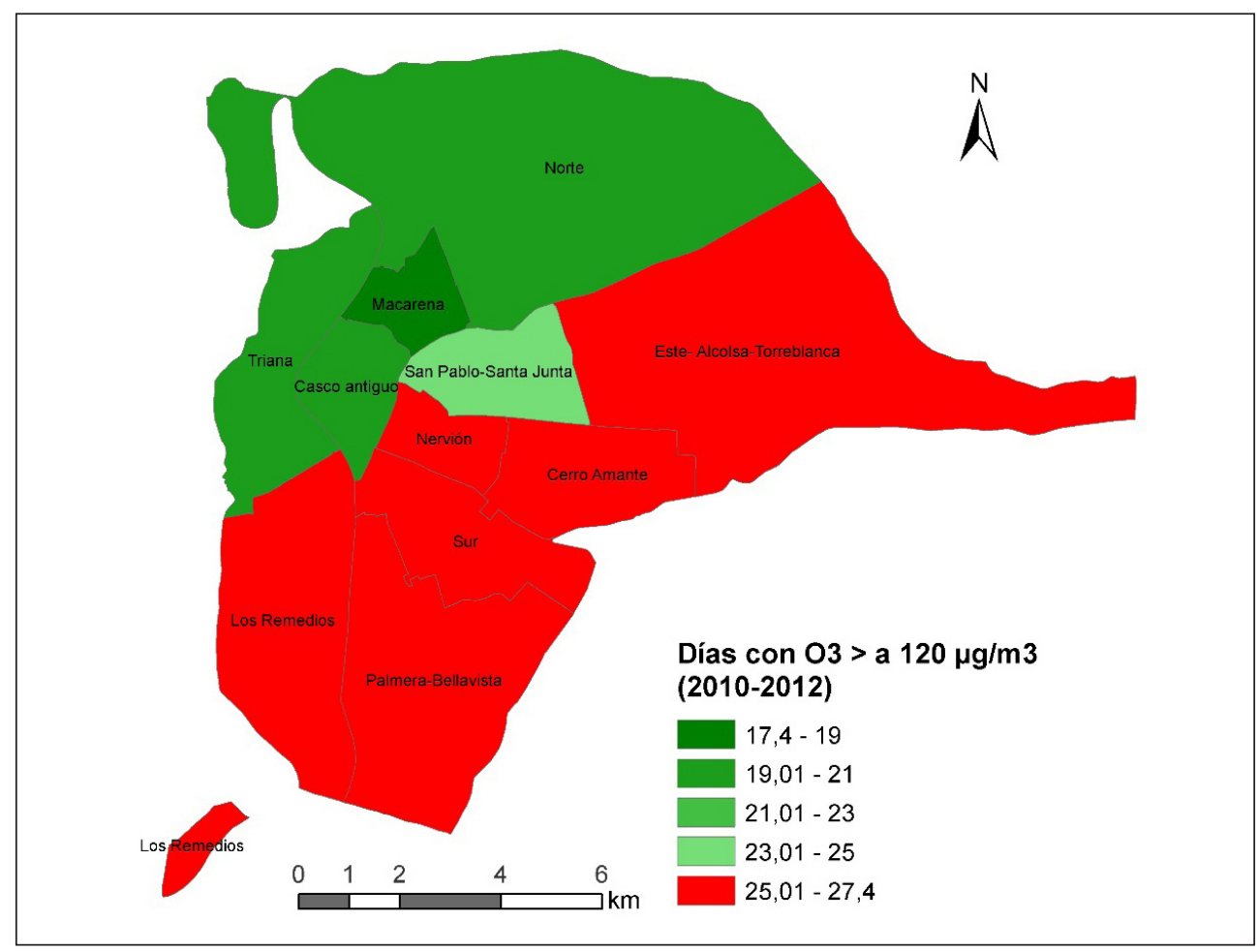

Figura 7. Número de días con $\mathrm{O}_{3}$ superiores a $120 \mu \mathrm{g} / \mathrm{m}^{3}$ en los distritos de Sevilla (2010-2012).

Fuente: Elaboración propia.

variables meteorológicas que son las responsables del aumento de su concentración atmosférica, como el de Vukovich et al., 2003, realizado para el corredor BaltimoreWashington, donde la temperatura del aire y la temperatura del punto de rocío son las variables implicadas en el aumento de ozono, aunque cuando utilizan datos exclusivamente de verano, las variables que más influyen son la cubierta nubosa y la dirección del viento. En Europa, coincidiendo con la ola de calor de 2003, Solberg et al. (2008) detectaron que, en varias ciudades de Francia, Alemania, Suiza y Austria, se alcanzaron los valores más altos de ozono desde finales de los años ochenta, y lo ponen en relación con una serie de realimentaciones positivas que tienen lugar entre las condiciones de tiempo y la subida de los niveles de ozono.

En los países que bordean el Mediterráneo, donde el clima es más cálido y existen elevadas tasas de radiación solar, en combinación con abundancia de precursores de ozono, se favorece más la generación de este contaminante. Así ocurre en Madrid y Sevilla, que son espacios de riesgo por contaminación de $\mathrm{O}_{3}$. En Madrid el porcentaje de 
superficie y población afectada es muy bajo (2,2\% y 0,2\% respectivamente), mientras que en Sevilla la situación es más inquietante puesto que el porcentaje de superficie expuesta alcanzaría la cifra del 58\% y el de población llegaría hasta el 56,5\%. También lo ha destacado Gerasopoulos et al. (2006) en la ciudad de Finokalia, en Creta. En la Península Ibérica sobresalen varios estudios; sobre el noreste de Portugal, Carvalho et al. (2010), caracterizaron las condiciones atmosféricas asociadas a episodios de alta concentración de ozono en la ciudad de Lamas d'Olo. Adame et al. (2010), investigaron las variaciones temporales del ozono en cuatro estaciones del suroeste de la Península Ibérica (El Arenosillo, Cartaya, Huelva y Valverde) y las superaciones de los umbrales establecidos por la UE. Las variaciones estacionales del ozono y su relación con la radiación, temperatura y dirección del viento fueron puestas de manifiesto en dos estaciones rurales, del centro-sur de España (Porzuna y Argamasilla) por Notario et al. (2012a). Sánchez et al. (2007) analizaron el intercambio vertical de ozono con mediciones realizadas en Segovia y Buitrago del Lozoya, detectando el desplazamiento del ozono y sus precursores desde Madrid hacia la Sierra de Guadarrama.

La distribución espacial del contaminante muestra que las zonas donde se superan los 25 días con concentraciones superiores a $120 \mu \mathrm{g} / \mathrm{m}^{3}$, son espacios alejados del centro urbano, zonas suburbanas y rurales, donde no existen suficientes inmisiones de óxido de nitrógeno que destruyan el ozono y éste acaba concentrándose en la atmosfera. Saavedra et al. (2012) constataron subidas de concentraciones de ozono en zonas suburbanas y rurales próximas a las grandes ciudades, en el noroeste de la Península Ibérica.

Los resultados alcanzados sirven para concienciar a los políticos, a los gestores públicos y a los ciudadanos de la deficiente calidad del aire urbano y de la necesidad de mejorar la red de calidad del aire, aumentando el número de estaciones y tomando medidas más estrictas y acordes con las recomendaciones de la OMS, encaminadas a mejorar los estándares ambientales y la calidad de vida de la población.

\section{Agradecimientos}

Este trabajo ha sido realizado en el marco del proyecto de investigación "Polución del aire, poblaciones vulnerables y salud: análisis de injusticias ambientales basados en geotecnologías" (Ref. CSO2014-55535-R), financiado por el Ministerio de Economía y Competitividad de España.

\section{Bibliografía}

Adame, J.A., Bolívar, J.P. y de la Morena, B. (2010). Surface ozone measurements in the Southwest of the Iberian Peninsula (Huelva, Spain). Environmental Science and Pollution Research, 17, pp. 355- 368. DOI: https://doi.org/10.1007/s11356-008-0098-9 
Ayuntamiento de Madrid (2020). Ozono y salud. Acceso web: https://www.madrid.es/portales/ munimadrid/es/Inicio/Servicios-sociales-y-salud/Salud/Publicaciones/Ozono-y-salud/ ?vgnextfmt=default\&vgnextoid=e4394bc638eaf310VgnVCM1000000b205a0aRCRD\&vgnextchannel=e6898fb9458fe410VgnVCM1000000b205a0aRCRD

Ballester, F., Tenías, J.M. y Pérez-Hoyos, S. (1999). Efectos de la contaminación atmosférica sobre la salud: una introducción. Revista Española de Salud Pública, vol. 73, 2, pp. 109-121. https://www.scielosp.org/article/resp/1999.v73n2/109-121/

Cañada, R., Moreno, A. y González, H. (2014). Modelado de la calidad del aire urbano. Un experimento metodológico con técnicas de interpolación espacial. Boletín de la Asociación de Geógrafos Españoles, 65, pp. 317-342. DOI: https://doi.org/10.21138/bage.1755

Cañada, R. y Moreno, A. (2017). El contraste intraurbano de la contaminación del aire por $\mathrm{NO}_{2}$ y $\mathrm{O}_{3}$ : estudio en grandes ciudades españolas con datos observados e interpolados con SIG. GeoFocus, no 19 , pp. 27-53. DOI: http://dx.doi.org/10.21138/GF.499

Cañada, R., Moreno, A. y Martínez, P. (2020). La amenaza de la polución atmosférica por PM $_{10}$, $\mathrm{NO}_{2}$ y $\mathrm{O}_{3}$ sobre la población de grandes ciudades españolas (Madrid, Barcelona y Sevilla): diagnóstico basado en modelos geoestadísticos y SIG. Geografía y Sistemas de Información Geográfica (GeoSIG), no 17, pp. 1-22. https://revistageosig.wixsite.com/geosig/geosig-17-2020

Carvalho, A., Monteiro, A., Ribeiro, I., Tchepel, O., Miranda, A.I., Borrego, C., Saavedra, C.S., Souto, J.A. y Casares, J.J. (2010). High ozone levels in the Northeast of Portugal: Analysis and characterization. Atmospheric Environment, 44, pp. 1020-1031. DOI: https://doi.org/10.1016/ j.atmosenv.2009.12.020

Dingenen, R., Dentener, F.J., Raes, F., Krol, M.C., Emberson, L., Cofala, J. (2009). The global impact of ozone on agricultural crop yields under current and future air quality legislation. Atmos. Environ., 43, pp. 604-618. DOI: https://doi.org/10.1016/j.atmosenv.2008.10.033

Díaz, J. y Linares, C. (2005). Salud ambiental y calidad de vida urbana. Educación para el desarrollo sostenible. Madrid: Ayuntamiento de Madrid, Dirección General de sostenibilidad y Agenda 21, 114 p.

Dueñas, C., Fernández, M.C., Cañete, S., Carretero, J. and E. Liger, E. (2002). Assessment of ozone variations and meteorological effects in an urban area in the Mediterranean coast. The Science of the Total Environment, vol. 299, pp. 97-113. DOI: https://doi.org/10.1016/S0048-9697 (02)00251-6

EEA (2014). Air quality in Europe - 2014 report. Luxembourg: Publications Office of the European Union.

EEA (2015). Air quality in Europe - 2014 report. Luxembourg: Publications Office of the European Union

EEA (2019). Air quality in Europe - 2019 report. Luxembourg: Publications Office of the European Union.

European Commission (2017). Special Eurobarometer 468: Attitudes of European citizens towards the environment (http://data.europa.eu/euodp/en/data/ dataset/S2156_88_1_468_ENG) accessed 20 June 2019.

Felzer, B.S., Cronin, T., Reilly, J.M., Melillo, J.M., Wang, X. (2007). Impacts of ozone on trees and crops. Impacts de l'ozone sur les arbres et les récoltes. Comptes Rendus Geoscience, 339, pp. 784-798. DOI: https://doi.org/10.1016/j.crte.2007.08.008 
Fishman, J., Creilson, J.K., Parker, P.A., Ainsworth, E.A., Vining, G.G., Szarka, J., Booker, F.L., Xu, $X$. (2010). An investigation of widespread ozone damage to the soybean crop in the upper Midwest determined from groundbased and satellite measurements. Atmospheric Environment, 44, pp. 2248-2256. DOI: https://doi.org/10.1016/j.atmosenv.2010.01.015

Gerasopoulos E., Kouvarakis, G., Vrekoussis, M., Donoussis, C., Mihalopoulos, N., Maria Kanakidou, M. (2006). Photochemical ozone production in the Eastern Mediterranean. Atmospheric Environment, 40, pp. 3057-3069. DOI: https://doi.org/10.1016/j.atmosenv.2005.12.061

Gottardini, E., Cristofori, A., Cristofolini, F., Ferretti, M. (2010). Variability of ozone concentration in a montane environment, northern Italy. Atmospheric Environment, 44, pp. 147-152. DOI: https://doi.org/10.1016/j.atmosenv.2009.10.017

Jerrett, M., Arain, A., Kanaroglou, P., Beckerman, B., Potoglou, D., Sahsuvaroglud, D., Amorrison, J. and Giovis, Ch. (2005). A review and evaluation of intraurban air pollution exposure models. Journal of Exposure Analysis and Environmental Epidemiology, 15, pp. 185-204. DOI: https://doi.org/10.1038/sj.jea.7500388

Loibl, W., Bolhar-Nordenkampf, H.R., Herman, F., Smidt, S. (2004). Modelling critical levels of ozone for the forested area of Austria. Modifications of the AOT40 concept. Env. Sci. Pollut. Res., 11 (3), pp. 171-180. DOI: https://doi.org/10.1007/BF02979672

Moral, F.J., Rebollo, F.J., Valiente, P., López, F. y Muñoz, A. (2012). Modelling ambient ozone in an urban area using an objective model and geostatistical algorithms. Atmospheric Environment, 63, pp. 86-93. DOI: https://doi.org/10.1016/j.atmosenv.2012.09.035

Notario, A., Díaz de Mera, Y., Aranda, A., Adame, J.A., Parra, A., Romero, E., Parra, J. y Muñoz, F. (2012). Surface ozone comparison conducted in two rural areas in central-southern Spain. Environmental Science and Pollution Research, 19 (1), pp. 186-200. DOI: https://doi.org/10.1007/ s11356-011-0551-z

Palacios, M., Kirchnner, F., Martilli, A., Clappier, A., Martín, F., Rodríguez, S. (2002). Summer ozone episodes in the Greater Madrid area. Analyzing the ozone response to abatement strategies by modelling. Atmospheric Environment., 36, pp. 5323-5333. DOI: https://doi.org/10.1016/ S1352-2310(02)00590-3

Saavedra, S., Rodríguez, A., Souto, J. A., Casares, J.J., Bermúdez, J.L. and B. Soto, B. (2012). Trends of Rural Tropospheric Ozone at the Northwest of the Iberian Peninsula. Scientific World Journal, DOI: $10.1100 / 2012 / 603034$

Sánchez, M.L., de Torre, B., García, M.A., Pérez, I. (2007). Ground-level ozone and ozone vertical profile measurements close to the foothills of the Guadarrama mountain range (Spain). Atmospheric Environment, 41, pp. 1302-1314. DOI: https://doi.org/10.1016/j.atmosenv.2006.09.047

Solberg, S., Hov, Ø., Søvde, A., Isaksen, ISA, Coddeville, P., De Backer. H., Forster, C., Orsolini, Y., Uhse, K. (2008). European surface ozone in the extreme summer 2003. Journal of Geophysical Research, 113, D07307. DOI: https://doi.org/10.1029/2007JD009098

Song, F., Shin, Y., Jusino-Atresino, R. y Gao, Y. (2011). Relationships among the springtime ground-level $\mathrm{NO}_{\mathrm{X}}, \mathrm{O}_{3}$ and $\mathrm{NO}_{3}$ in the vicinity of highways in the US East Coast. Atmospheric Pollution Research, 2, pp. 374-383. DOI: https://doi.org/10.5094/APR.2011.042

Vukovich, F.M. y Sherwell, J. (2003). An examination of the relationship between certain meteorological parameters and surface ozone variations in the Baltimore-Washington corridor. Atmospheric Environment, 37, pp. 971-981. DOI: https://doi.org/10.1016/S1352-2310(02)00994-9 